\section{Miller Fisher syndrome with sinus arrest}

\author{
Nobuko Shiraiwa, ${ }^{1,2}$ Mitsumasa \\ Umesawa,2,3 Sachiko Hoshino, ${ }^{2}$ \\ Tsuyoshi Enomoto, 4 Susumu Kusunoki, 5 \\ Akira Tamaoka, ${ }^{6}$ Norio Ohkoshi1 \\ 1Course of Neurology, Department of \\ Health, Faculty of Health Sciences, \\ Tsukuba University of Technology; \\ 2Department of Neurology, Tsukuba \\ Memorial Hospital; ${ }^{3}$ Department of \\ Public Health, School of Medicine, \\ Dokkyo Medical University; \\ ${ }^{4}$ Department of Cardiology, Tsukuba \\ Memorial Hospital; 5Department of \\ Neurology, Faculty of Medicine, Kindai \\ University; 6Department of Neurology, \\ Graduate School of Comprehensive \\ Human Science, University of Tsukuba, \\ Tsukuba, Japan
}

\section{Abstract}

Dysautonomia in Guillain-Barre syndrome (GBS) rarely causes serious cardiovascular complications, such as sinus arrest. Miller Fisher syndrome (MFS) is recognized as a variant of GBS. There have been few reports regarding the association between MFS and dysautonomia. We describe a case of a 68-year-old man with ophthalmoplegia, bulbar palsy, truncal ataxia, and areflexia. He was diagnosed with MFS because he exhibited the classical clinical triad and had elevated serum antiGQ1b immunoglobulin G levels. A magnetic resonance imaging scan of his head was normal. His 24-hour Holter recording showed sinus arrest. He was treated with intravenous immunoglobulin, whereupon his symptoms gradually improved. This included the sinus arrest, which was considered a symptom of dysautonomia in MFS. Therefore, clinicians should be mindful of dysautonomia not only in GBS patients, but also in cases of MFS.

\section{Introduction}

Dysautonomia is present in approximately two-third of patients with GuillainBarre syndrome (GBS). ${ }^{1}$ It results in a wide range of symptoms, including various cardiac arrhythmias.

Although dysautonomia is usually of minor clinical importance, it may cause lifethreatening cardiovascular complications. Serious arrhythmias have been found in 7-
$34 \%$ of GBS patients. Recent studies indicate that these complications are not limited to severely disabled patients who require mechanical ventilation, but may also affect those with milder forms of the condition, who do not require artificial ventilation, and are able to walk more than 5 meters. ${ }^{2,3}$

Miller Fisher syndrome (MFS) is recognized as a variant of GBS. It is a relatively rare condition that is characterized by the acute onset of ophthalmoplegia, ataxia, and areflexia or hyporeflexia.4,5 An association between MFS and autonomic dysfunction has been previously reported, with cardiovascular complications being rare.6,7 The present case report describes sinus arrest in a patient with MFS.

\section{Case Report}

A 68-year-old man had an acute onset of truncal ataxia, dysarthria, and diplopia in March 2009. He developed dysphagia the next day and underwent a magnetic resonance imaging (MRI) scan, which was normal. He presented to our neurology outpatient department with truncal ataxia, opthalmoplegia, and areflexia five days later.

Upon admission, he had a normal blood pressure of 136/96 $\mathrm{mmHg}$, a regular heart rate of 98 beats per minute (bpm), and a peripheral capillary oxygen saturation (SpO2) of $97 \%$ in room air. Neurological examination revealed mydriasis, absent pupillary light reflex bilaterally, total ophthalmoplegia with both eyes fixed centrally, bulbar palsy with nasal voice, dysphagia, absent pharyngeal reflex, and absent deep tendon reflexes. There was no limb weakness. Finger-to nose and heel-to-shin tests showed no dysmetria or decomposition. Romberg's test and Mann's test were both positive. He was unable to walk unaided due to the truncal ataxia. There were no sensory abnormalities, orthostatic hypotension, and dysfunction of the bladder and bowel.

His blood count and serum biochemical parameters were normal. Antinuclear, antideoxyribonucleic acid (DNA), and antiacetylcholine receptor (Ach R) antibodies were all negative. Vitamin B1 levels were normal (46.3 ng/mL). Cerebrospinal fluid analysis was normal with a cell count of $1 / \mu \mathrm{L}$ (mononuclear leukocytes), protein level of $33 \mathrm{mg} / \mathrm{dl}$, glucose level of $66 \mathrm{mg} / \mathrm{dl}$ (serum glucose $97 \mathrm{mg} / \mathrm{dl}$ ), and an IgG index of 0.52. However, he tested positive for serum anti-GQ1b IgG, an autoantigenic antiganglioside antibody. His repeated head MRI and chest X-rays were normal.

Given his presentation with acute onset total ophthalmoplegia, ataxia, and areflexia, he was diagnosed with MFS. He had a
Correspondence: Nobuko Shiraiwa, Course of Neurology, Department of Health, Faculty of Health Sciences, Tsukuba University of Technology, 4-12-7 Kasuga, Tsukuba, Ibaraki 305-8521, Japan.

E-mail: shiraiwa@xa2.so-net.ne.jp

Key words: Miller Fisher syndrome, GuillainBarre syndrome, Dysautonomia, Sinus arrest.

Conflicts of interest: the authors declare no potential conflicts of interest.

Acknowledgments: we would like to thank Editage (www.editage.jp) for English language editing.

Received for publication: 14 July 2017. Accepted for publication: 23 July 2017.

This work is licensed under a Creative Commons Attribution NonCommercial 4.0 License (CC BY-NC 4.0).

C Copyright N. Shiraiwa, et al., 2017 Licensee PAGEPress, Italy

Neurology International 2017; 9:7312

doi:10.4081/ni.2017.7312

Functional Systems Score (FSS) of 3 upon admission. Treatment with intravenous immunoglobulin (IVIG; $0.4 \mathrm{~g} / \mathrm{Kg}$ body weight/day for 5 days) was initiated on day six. He underwent 24-hour Holter recording on day seven because he had exhibited bradycardia (heart rate under $40 \mathrm{bpm}$ ), sinus arrest with the $\mathrm{P}$ wave falling off, and a 2.94-second duration of the greatest R-R interval (Figure 1). There were five pauses daily. The 24-hour Holter recording also displayed atrioventricular dissociation with junctional escape beats. After commencing IVIG therapy, his bulbar palsy improved first, followed by the internal ophthalmoplegia and truncal ataxia. In addition, a repeat 24-hour Holter recording on day 19 showed that the sinus arrest had resolved. He was discharged with an FSS of 2 on day 32. Approximately two months after the onset of symptoms, the diplopia secondary to the ophthalmoplegia had resolved.

\section{Discussion}

The present report described a classic case of MFS defined by the acute onset of ophthalmoplegia, ataxia, and areflexia, with positive detection of serum anti-GQ1bIgG antibody. The patient showed sinus arrest early after the onset of his symptoms. The sinus arrest, together with the other symptoms, improved soon after he had been 
commenced IVIG therapy. There was no need for cardiac pacemaker insertion.

Similarly to GBS, cardiovascular complications are rarely reported in MFS and are considered to be a complication of dysautonomia.6,7 Kuzumoto et al. showed an association between autonomic test abnormality and anti-GQ1b antibody in MFS. ${ }^{8}$ Recent studies on GBS indicate that these complications are not only limited to severely disabled patients who require mechanical ventilation, but also affect those with milder forms of the condition, who do not require artificial ventilation and are able to walk more than 5 meters. 2,3 The degree of motor impairment could not predict the occurrence of serious bradyarrythmias, including sinus arrest. Early recognition of such life-threatening events is important so that appropriate preventive measures, such as the insertion of a cardiac pacemaker, can be promptly taken.

\section{Conclusions}

This report has shown that rare lifethreatening cardiovascular complications may occur not only in GBS, but also in MFS.

\section{References}

1. Hartung HP, Pollard JD, Harvey GK, et al. Immunopathogenesis and treatment of the Guillain-Barre syndrome-Part I. Muscle Nerve 1995;18:137-53.

2. Flachenecker P, Lem K, Mullges W, et al. Detection of serious bradyarrhythmias in Guillain-Barre syndrome: sensitivity and specificity of the 24-hour
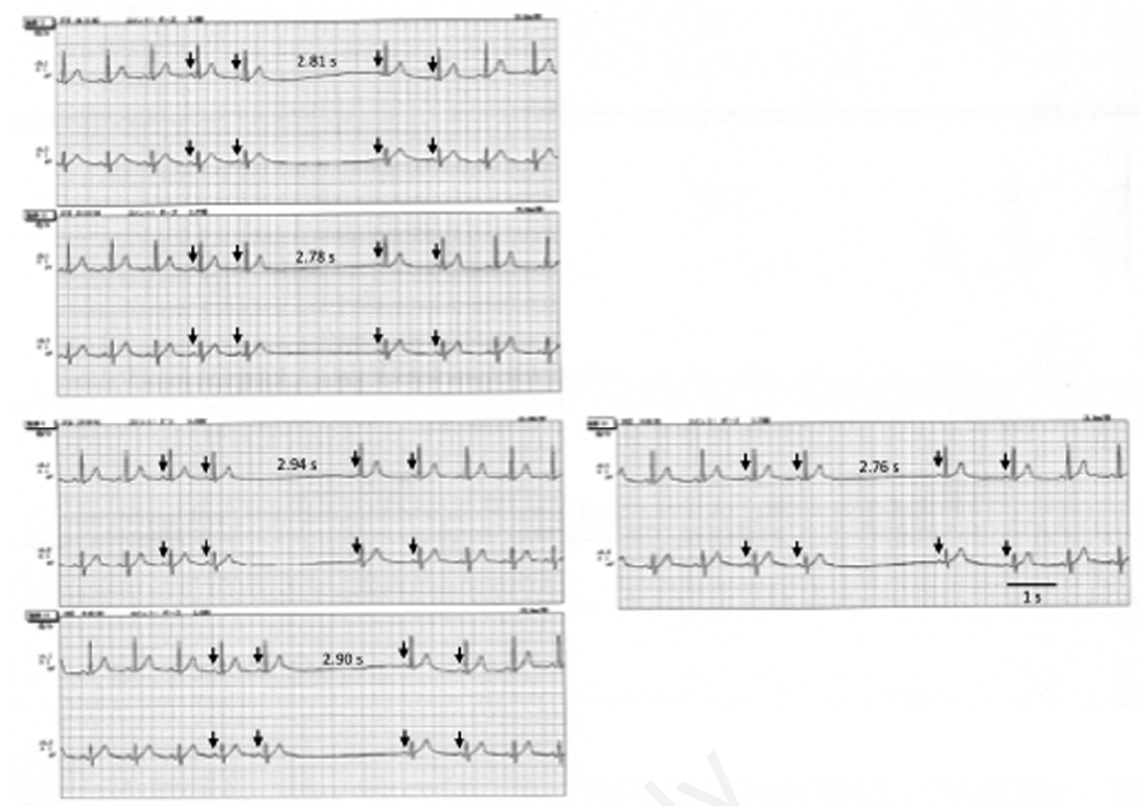

Figure 1. Holter recording on day seven displaying sinus arrest with $P$ wave (arrow) falling off, and 2.94 seconds at the greatest $R-R$ interval. There were five pauses daily.

heart rate power spectrum. Clin Auton Res 2000;10:185-91.

3. Flachenecker P, Mullges W, Wermuth P, et al. Eyeball pressure testing in the evaluation of serious bradyarrhythmias in Guillain-Barre syndrome. Neurology 1996;47:102-8.

4. Fisher M. An unusual variant of acute idiopathic polyneuritis (syndrome of ophthalmoplegia, ataxia and areflexia). New Engl J Med 1956;255:55-65.

5. Wakerley BR, Uncini A, Yuki N. Guillain-Barre and Miller Fisher syndrome-new diagnostic classification. Nat Rev Neurol 2014;10:537-44.

6. Kordouni M, Jibrini M, Siddiqui MA.
Long-term transvenous temporary pacing with active fixation bipolar lead in the management of severe autonomic dysfunction in Miller Fisher syndrome: a case report. Int $\mathrm{J}$ Cardiol 2007;117:e10-2.

7. Osada O, Iwasaki A, Kusunoki S. Fisher syndrome with 2:1 atrioventricular block supposed by brain's regulating failure of autonomic nervous system. A case report. Neurol Med 2016;84:31621.

8. Kuzumoto Y, Shioyama M, Kihara M, Kusunoki S. Abnormal sudomotor axon reflex and antiganglioside antibodies. Muscle Nerve 2006;33:828-9. 